\title{
Optimal Universal and Categorical Benefit Provision with Classification Errors and Imperfect Enforcement.*
}

\author{
Sean Slack ${ }^{\dagger}$ David Ulph
}

June 2016

\begin{abstract}
We determine the optimal combination of a universal benefit and categorical benefit when individuals differ in their ability to work and, if able to work, their productivity. The categorical benefit is conditioned ex-ante on applicants being unable to work and ex-post on recipients not working. The awards test makes Type I/II errors. If the expost condition is (i) not enforced the optimal categorical benefit is positive only if the awards test has discriminatory power, whilst maximum welfare falls with both error propensities; but if (ii) fully enforced the optimal categorical benefit is positive always and maximum welfare can increase with the Type II error propensity.
\end{abstract}

Keywords: Categorical Benefit; Classification Errors, Universal Benefit; Optimal Taxation

JEL Classification Numbers: D63; H21; H53; I31; I38

${ }^{*}$ Financial support from the AXA Research Fund is gratefully acknowledged. We also acknowledge SIRE for a conference presenter grant to present an earlier version of this paper at PET 14 Seatle, July 11-13, 2014. Finally, we wish to thank an anonymous referee for their highly detailed and constructive feedback.

${ }^{\dagger}$ School of Economics \& Finance, University of St. Andrews, Castlecliffe, The Scores, St. Andrews, Fife, KY16 9AR, Scotland, U.K. Email: sean.ses34@gmail.com

${ }^{\ddagger}$ School of Economics \& Finance, University of St. Andrews, Castlecliffe, The Scores, St. Andrews, Fife, KY16 9AR, Scotland, U.K. Email: du1@st-andrews.ac.uk 


\section{Introduction}

Partial universal welfare systems can be defined as those which (i) provide an unconditional universal benefit to all individuals in society; but also (ii) allow for additional targeted assistance to those judged by the policymaker to be most in need. ${ }^{1}$ Whilst targeted transfers play the prominent role in modern welfare systems, much debate surrounds the extent to which they reach those in need, for example due to misclassifications or non-take-up (Currie, 2006; Moffitt, 1983). This has led numerous authors to discuss proposals for partial universal programmes (Callan et al., 1999; Van Parijs, 2004). In particular, Atkinson (1995) analyses universal benefit provision within a linear income tax model, where a fraction of the population may be involuntarily unemployed and may also receive a categorical benefit.

Categorical benefits are an important form of targeting and have two salient features: ${ }^{2}$

(i) Double Conditionality. Categorical benefits are typically conditioned in two dimensions: ex-ante an applicant must belong to some categorical group and satisfy other initial conditions to be awarded the benefit; whilst ex-post a recipient must comply with certain behavioural criteria. For example, disability benefits are often conditioned exante on applicants having a disability that significantly affects their ability to work; and ex-post on some form of work restriction (Salanié, 2002; Jacquet, 2006).

(ii) Imperfect Enforcement. Both dimensions of conditionality may be imperfectly enforced. First, ex-ante conditionality may be violated by classification errors of Type I (false rejection) and Type II (false award) in the screening process. Again, disability benefits provide a good example because certain medical conditions (e.g. musculoskeletal disease, mental illness) can be difficult to verify, as can be their impact on functional criteria. ${ }^{3}$ Second, ex-post conditionality may be violated because the authorities fail to detect all recipients who break the requirements; whilst the sanctions imposed if successfully detected are insufficient to deter such behaviour (Fuller et al., 2015).

Note that in many cases an individual who is ex-ante eligible will automatically satisfy ex-post requirements. For example, an individual who is truly unable to work due to disability and awarded benefits on this basis, will automatically satisfy any ex-post no-work requirement.

\footnotetext{
${ }^{1}$ The universal benefit may also be referred to as a basic income or demogrant (Van Parijs, 2004).

${ }^{2}$ Many types of benefit may condition on categorical status; these may fall under social assistance (e.g. Supplementary Security Income in the U.S.); social insurance (e.g. Social Security Disability Insurance in the U.S.); or instead be strictly categorical (i.e. the amount awarded to legitimate recipients is independent of means or insurance contributions). The model presented in this paper is most related to the latter case.

${ }^{3} \mathrm{~A}$ number of studies estimate error propensities for real-world programmes (Benitez-Silva et al., 2004; Nagi, 1969; Duclos, 1995).
} 
To this extent, Type II errors will be the source of ex-post enforcement issues.

In the context of partial universal programmes three central questions therefore arise:

1. How does the propensity of the awards technology to make Type I and Type II errors affect the optimal levels of a universal benefit and categorical benefit, respectively? In particular, are there conditions under which it is optimal to choose either a purely universal or purely categorical system, as opposed to a partial system?

2. How do these error propensities affect the resulting level of social welfare?

3. How do the answers to both of the above questions depend on how well the ex-post conditionality is enforced?

The contribution of this paper is to address the three questions raised above within a framework that allows for a systematic comparison of how the answer to the first two questions depends on how well the ex-post conditionality is enforced. We first discuss the key literature related to this paper and then proceed to outline our framework and key results.

Relevant Literature. The existing literature on imperfectly targeted benefits has tended to focus on the first question. Two key studies - Parsons (1996) and Salanié (2002) - determine the optimal benefit structure when an exogenous tagging technology makes two-sided classification errors in identifying unable individuals. Whilst these papers make differing assumptions on the ex-post conditions placed on tagged individuals, other differences in the modelling frameworks do not facilitate direct comparison. In Parsons (1996) individuals are ex-ante identical but may, with some exogenous probability, become unable to work. Individual preferences are quasilinear (linear in leisure). Parsons demonstrates that the standard tagging outcome ${ }^{4}$ can be improved upon by a system which pays tagged able individuals to work, provided these individuals can be incentivised to work for less than their marginal product. The assumption is thus made that tagged individuals are allowed to work. Contrastingly, Salanié (2002) adopts the opposite assumption in a more general framework where individuals who are able to work differ continuously in their productivity. He demonstrates that it is optimal to award tagged individuals a higher benefit. The surprising feature of the analysis is that application decisions are not modelled. Instead, a fraction of the able subpopulation - corresponding to the Type II error propensity - are simply tagged and not allowed to work. However, this must be incentive incompatible for higher productivity individuals who would rather receive the lower untagged benefit and be allowed to work.

\footnotetext{
${ }^{4}$ The standard tagging outcome refers to the Akerlof (1978) case, modified to incorporate Type II errrors.
} 
A number of papers analyse categorical transfers in the optimal income tax framework originating from Mirrlees (1971): see Immonen et al. (1998); Kaplow (2007); Viard (2001a,b). In these papers the planner partitions society into disjoint categorical groups, each with its own ability/productivity distribution. It then chooses the tax/benefit schedule for each group (or a common linear income tax and group specific categorical transfers).

Our framework. We consider a framework where a fraction of the population faces a zeroquantity constraint on labour and are thus unable to work, whilst the remaining fraction are able to work and differ continuously in their productivity. The government operates a tax/benefit system comprising (i) a constant marginal tax rate on earned income; (ii) a tax-free universal benefit $(B)$ received unconditionally by all individuals; and (iii) a tax-free categorical benefit $(C)$ that is targeted at those who are unable to work. We undertake the main analysis with a fixed benefit budget. Following this, we demonstrate that many of the key results continue to hold in an optimal linear income tax model. Starting with a fixed budget greatly simplifies the exposition and allows us to pin down more precisely the intuition for the conditions under which it is optimal to provide a categorical benefit. ${ }^{5}$

The categorical benefit is conditioned ex-ante on an applicant being unable to work; and expost on a recipient not working. However, the exogenous awards test makes Type I and Type II errors. The test has perfect discriminatory power if there is a zero propensity for either error type; no discriminatory power if unable and able applicants are awarded the benefit with the same probability; and some discriminatory power in all intermediate cases.

Applications for the categorical benefit are taken to be costless in terms of money, stigma and time. ${ }^{6}$ This eliminates the direct dependence of application decisions on classification error propensities (Jacquet, 2006, 2014). We assume that no checks or penalties are in place for an individual who is incorrectly awarded the benefit but subsequently does not work. There are two reasons for this: (i) such behaviour is highly difficult to detect as the able recipient does not reveal their true type through working (Yaniv, 1986); whilst (ii) in reality applicants may be uncertain of their own ex-ante eligibility. Accordingly, some lower productivity able individuals will always apply for the categorical benefit and, if awarded it, not work.

However, the application decisions of higher productivity able individuals will intuitively depend on how effectively the ex-post no-work condition is enforced. We systematically compare two discrete alternative enforcement regimes: No Enforcement and Full Enforcement. Under No Enforcement there are no mechanisms in place to detect and sanction any

\footnotetext{
${ }^{5}$ Note that some countries such as the U.K. now operate a welfare cap on (forecasted) benefit expenditures.

${ }^{6}$ On these issues see Currie (2006); Duclos (1995); and Moffitt (1983).
} 
able recipient of the categorical benefit who works: all able individuals therefore apply. Contrastingly, under Full Enforcement it is assumed that the probability of detection and the penalty regime in place are sufficiently tough that no able recipient of the categorical benefit will choose to work: consequently, only able individuals of sufficiently low productivity (i.e. low opportunity cost of not working) will apply.

The government chooses the universal and categorical benefits $(B, C)$ to maximise a strictly utilitarian social welfare function subject to its budget constraint. In the baseline case where the benefit authority can perfectly discriminate between unable and able applicants it will always be optimal to set $C>0$, but $B>0$ only if the benefit budget exceeds that which, if spent exclusively on categorical transfers, eliminates inequality in the average smvi between the unable and able subpopulations. The result that categorical transfers should be set to eliminate between-group inequality across categorical groups is well established (Beath et al., 1988; Immonen et al., 1998; Viard, 2001a,b). However, with Type I and Type II errors between-group inequality in the average smvi will persist at the optimum.

The major conclusions of this paper (described for the fixed budget analysis) are:

- Under a No Enforcement regime:

1. It is optimal to set $C>0$ only if the awards test has at least some discriminatory power. If the test has no discriminatory power a targeted system provides both subpopulations with, on average, the same benefit income and so does no better between-group than a pure universal system, but also does worse within-group. In an optimum with $C>0$ we have $B>0$ if Type I errors occur so as to avoid some unable individuals having no income; but otherwise $B>0$ only if the budget is sufficiently large.

2. Maximum social welfare decreases with Type I and Type II error propensities. This implies that while society will benefit from reforms that increase test accuracy ${ }^{7}$, changes that simply shift the liability threshold - i.e. make the test more stringent/lenient - will involve a trade-off between error types and have an overall ambiguous welfare effect. ${ }^{8}$

- Under a Full Enforcement regime:

1. It is optimal to set $C>0$ for all levels of discriminatory power. Even when the test has no discriminatory power a targeted system provides the unable with, on average, more benefit income than the able. Furthermore, Type II errors redistribute within the able subpopulation because only low productivity individuals apply. If Type I errors occur

\footnotetext{
${ }^{7}$ In reality, these gains must be set against any costs of investing in test accuracy.

${ }^{8}$ Goodin (1985) refers to such changes in the liability threshold as 'shifts in test-bias'.
} 
with positive propensity then it is always optimal to set $B>0$.

2. Whilst maximum social welfare decreases with the Type I error propensity, there are conditions under which it can increase with the Type II error propensity. Where these conditions are satisfied, the implication is that changes which lower the liability threshold - i.e. make the test more lenient - will reduce Type I errors but increase Type II errors and so can be welfare increasing.

This paper is structured as follows. Section 2 sets out the model; Section 3 presents the main analysis; Section 4 extends the analysis to an optimal tax framework; and finally Section 5 concludes. All proofs are situated in the supplementary online appendix.

\section{The Model}

\subsection{Background: Individual Behaviour}

Individuals have identical preferences given by the utility function $u(x, l)$, where $x \geq 0$ denotes consumption and $l \in[0,1]$ denotes leisure respectively. The standard assumptions apply: $u$ is continuously differentiable, strictly increasing in both arguments $\left(u_{x}>0, u_{l}>0\right)$ and strictly concave $\left(u_{x x}<0 ; u_{l l}<0\right.$ and $\left.u_{x x} u_{l l}-u_{x l}^{2}>0\right)$. We also assume that leisure is normal $\left(u_{l} u_{x x}-u_{x} u_{x l}<0\right)$ and that:

$$
\lim _{x \rightarrow 0} u_{x}(x, l)=+\infty
$$

An individual with net wage $\omega \geq 0$ and unearned income $M \geq 0$ chooses labour supply, $H \in$ $[0,1]$, to maximise utility. Optimal labour supply $\left(H^{*}\right)$ and indirect utility $(v)$ satisfy:

$$
\begin{aligned}
H^{*}(\omega, M) & \equiv \arg \max _{0 \leq H \leq 1} u(\omega H+M, 1-H) \\
v(\omega, M) & \equiv \max _{0 \leq H \leq 1} u(\omega H+M, 1-H)=u\left(\omega H^{*}+M, 1-H^{*}\right)
\end{aligned}
$$

Let $\bar{\omega}(M) \equiv u_{l}(M, 1) / u_{x}(M, 1)$ be the reservation wage satisfying $H^{*}=0 \forall \omega \leq \bar{\omega}(M)$, whilst $H^{*}>0 \forall \omega>\bar{\omega}(M)$ and $\omega=u_{l}\left(\omega H^{*}+M, 1-H^{*}\right) / u_{x}\left(\omega H^{*}+M, 1-H^{*}\right)$. By the normality of leisure $\bar{\omega}^{\prime}(M)>0$ whilst $H_{M}^{*}(\omega, M)<0 \forall \omega>\bar{\omega}(M)$.

Finally, $\forall \omega \geq 0$ we have $v_{\omega}(\omega, M)=v_{M}(\omega, M) H^{*}(\omega, M)$ (i.e. Roy's identity) and so: 


$$
\forall \omega>\bar{\omega}(M): v_{\omega M}(\omega, M)<0 \Rightarrow v_{M}(\omega, M)<v_{M}[\bar{\omega}(M), M]=u_{x}(M, 1)
$$

Over this range of net wages the the marginal indirect utility of unearned income is thus a strictly decreasing function of the net wage.

\subsection{The Population and Tax-Benefit System}

In a population of size 1 the fraction $\theta \in(0,1)$ of individuals face a zero quantity constraint on labour supply and are thus unable to work. The remaining fraction $(1-\theta)$ of individuals are able to work but differ in their productivity, $n \geq 0$.

There is a tax-benefit system comprising four elements: (i) a linear income tax, $\tau \in(0,1)$; (ii) a tax-free universal benefit, $B \geq 0$, paid automatically to everyone ${ }^{9}$; (iii) a tax-free categorical benefit, $C \geq 0$, that is targeted - potentially imperfectly - at those who are unable to work and awarded in addition to $B$; and (iv) a fixed benefit budget, $\beta>0$.

We take $\tau$ as exogenously given. Productivities are captured through the net wage, $\omega \equiv n(1-$ $\tau)$, as distributed with density function $f(\omega)$, where $f(\omega)>0 \forall \omega \geq 0$ and $\int_{0}^{\infty} f(\omega) d \omega=1$. The associated distribution function is $F(\omega)=\int_{0}^{\omega} f(z) d z$, where $0 \leq F(\omega) \leq 1$.

$C$ must be applied for and applications are taken to be costless in terms of money, stigma and time. In line with real-world categorical transfers, $C$ is conditioned in two dimensions: ex-ante an applicant must be unable to work to be awarded the benefit; whilst ex-post a recipient must not work when receiving the benefit. However, ex-ante conditionality may be violated because the test awarding $C$ may make Type I (false rejection) and Type II (false award) classification errors with exogenous propensities $p_{i} ; i \in\{I, I I\}$. We assume:

$$
p_{I}+p_{I I} \leq 1
$$

We say that the test awarding $C$ has perfect discriminatory power if $p_{I}=p_{I I}=0$; some discriminatory power if $0<p_{I}+p_{I I}<1$; but no discriminatory power if $p_{I}+p_{I I}=1$.

The assumption that applications for $C$ are costless renders individual welfare in the rejected state the same as that from having not applied. This eliminates the direct dependence of individual application decisions on $p_{I}$ and $p_{I I}$ (Jacquet, 2006, 2014). An unable individual will therefore always choose to apply for $C$. If $p_{I I}=0$ ex-post conditionality is automatically satisfied because all recipients of $C$ are unable to work. Contrastingly, $p_{I I}>0$ generates a

\footnotetext{
${ }^{9}$ With just these first two elements, we would have effectively a simple negative income tax system.
} 
host of enforcement issues because whether or not an able individual will choose to apply for $C$ will depend on the mechanisms in place to detect ineligible recipients. First, it is assumed that there are no checks or penalties in place for an able individual who applies for $C$ and, if awarded it, does not work. There are two reasons for this: (i) such behaviour is highly difficult to detect because the able recipient exactly mimics the unable recipient (Yaniv, 1986); and (ii) it is not immediately clear that such behaviour is 'fraudulent' because an individual may be unsure of their own eligibility upon applying. Given this assumption, all able individuals with $\omega<\bar{\omega}(B+C)$ will certainly apply for $C$, where $\bar{\omega}(B+C)>\bar{\omega}(B) \forall C>0$.

Second, which individuals commanding $\bar{\omega}(B+C)<\omega$ will choose to apply for $C$ will depend on how effectively the ex-post no-work condition is enforced. In this regard, we analyse two binary enforcement assumptions: (i) No Enforcement and (ii) Full Enforcement.

\subsubsection{No Enforcement}

In this first case we assume that there are no mechanisms in place to detect and sanction a recipient of $C$ who works. Accordingly, all able individuals will choose to apply for $C$. Of those awarded it, individuals with $\omega \leq \bar{\omega}(B+C)$ will not work; whilst individuals with $\omega>\bar{\omega}(B+C)$ will work.

\subsubsection{Full Enforcement}

In this second case, we alternatively assume that there are totally effective mechanisms in place that fully deter any able individual from working whilst receiving $C$. As under No Enforcement, all those with $\omega \leq \bar{\omega}(B+C)$ will apply for $C$ because they would choose to do so were there no restrictions on labour supply. However, those with $\omega>\bar{\omega}(B+C)$ will only choose to apply for $C$ if $\omega<\overline{\bar{\omega}}(B, C)$, where:

$$
v[\overline{\bar{\omega}}(B, C), B] \equiv u(B+C, 1)
$$

The function $\overline{\bar{\omega}}$ is thus the critical net wage at which an individual is indifferent between (i) receiving $C$ but facing a fully-enforced zero quantity constraint on labour; and (ii) not receiving $C$ but being unrestricted in their labour supply. One can readily show that:

$$
\overline{\bar{\omega}}_{C}>\overline{\bar{\omega}}_{B}>0
$$

Finally, since $v[\bar{\omega}(B+C), B+C] \equiv u(B+C, 1) \equiv v[\overline{\bar{\omega}}(B, C), B]$, it must hold that $\bar{\omega}(B+C)<$ 
$\overline{\bar{\omega}}(B, C) \forall C>0$; where $\lim _{C \rightarrow 0} \overline{\bar{\omega}}(B, C)=\bar{\omega}(B)$.

Policy Constrained Individuals. Notice that the only recipients of $C$ for whom the fully enforced zero quantity constraint binds are those with $\bar{\omega}(B+C)<\omega \leq \overline{\bar{\omega}}(B, C) .{ }^{10}$

\section{Analysis}

We proceed to the main the analysis. The key questions we wish to answer are:

1. For what levels of discriminatory power would it be optimal to adopt a (i) pure universal system $(B>0, C=0)$, (ii) partial universal system $(B>0, C>0)$, or (iii) pure targeted system $(B=0, C>0)$ ?

2. How does maximum social welfare change with the classification error propensities?

3. How do the answers to these questions differ across the two enforcement regimes?

We throughout adopt a strictly utilitarian social welfare function.

\subsection{Perfect Discrimination (Baseline Case)}

When the test administering $C$ has perfect discriminatory power, social welfare is:

$$
W^{P}(B, C ; \theta)=\theta u(B+C, 1)+(1-\theta) \int_{0}^{\infty} v(\omega, B) d F(\omega)
$$

Given the fixed budget $\beta$ available for benefit expenditure, the optimisation problem is:

$$
\max _{B, C} W^{P}(B, C ; \beta, \theta) \text { s.t. } B+\theta C=\beta, B \geq 0, C \geq 0
$$

The budget constraint binds because social welfare can always be raised through paying everyone a higher $B$. Letting $\hat{B}^{P}(\beta, \theta)$ and $\hat{C}^{P}(\beta, \theta)$ denote the optimal choices, we have:

\section{Proposition 1.}

\footnotetext{
${ }^{10}$ It is important to stress that the issue of ex-post enforcement is a rich one and properly modelling this outside the extreme cases of No Enforcement and Full Enforcement is a non-trivial matter: one must consider (i) information sharing between the tax authority and benefit authority; (ii) the penalties imposed upon an individual who is detected breaking the ex-post conditions; and (iii) individual attitudes to risk. These points are all related. In reality, information sharing/cross-checking technologies are likely to be imperfect (Fuller et al., 2015). If the penalty imposed on fraudulent recipients is proportional to the benefit size then the variance in benefit income will play an important role in the risk individuals face.
} 
(i) $\hat{C}^{P}(\beta, \theta)>0$

(ii) The optimal benefits satisfy:

$$
\int_{0}^{\infty} v_{M}\left(\omega, \hat{B}^{P}\right) d F(\omega) \leq u_{x}\left(\hat{B}^{P}+\hat{C}^{P}, 1\right) \quad ; \quad \hat{B}^{P} \geq 0
$$

where the pair of inequalities hold with complementary slackness. It follows that $\hat{B}^{P}(\beta, \theta)>0$ only if $\beta>\bar{\beta}$, where $\bar{\beta}^{P}$ is implicitly defined by:

$$
\int_{0}^{\infty} v_{M}(\omega, 0) d F(\omega) \equiv u_{x}\left(\frac{\bar{\beta}^{P}}{\theta}, 1\right)
$$

Proof: See supplementary online Appendix.

Proposition 1 states that (i) it is always optimal to provide a categorical benefit; but (ii) it is only optimal to provide a universal benefit if the benefit budget exceeds a critical level which, if spent only on categorical transfers, would eliminate inequality in the average social marginal value of income (smvi) between the unable and able subpopulations. The result that categorical benefits should be set so as to equate the average smvi across categorical groups is well-documented (Beath et al., 1988; Immonen et al., 1998; Viard, 2001a,b).

\subsection{Imperfect Discrimination: No Enforcement}

With no mechanisms in place to enforce the condition that recipients of $C$ do not work, all able individuals apply for $C$. Social welfare is therefore now given by:

$$
\begin{aligned}
W^{N}\left(B, C ; \theta, p_{I}, p_{I I}\right) & =\theta\left\{\left(1-p_{I}\right) u(B+C, 1)+p_{I} u(B, 1)\right\} \\
& +(1-\theta)\left\{\begin{array}{c}
p_{I I}\left\langle F[\bar{\omega}(B+C)] u(B+C, 1)+\int_{\bar{\omega}(B+C)}^{\infty} v(\omega, B+C) d F(\omega)\right\rangle \\
+\left(1-p_{I I}\right)\left\langle F[\bar{\omega}(B)] u(B, 1)+\int_{\bar{\omega}(B)}^{\infty} v(\omega, B) d F(\omega)\right\rangle
\end{array}\right\}
\end{aligned}
$$

The first line on the right side of (11) is the aggregate welfare of unable individuals: Type I errors introduce horizontal inequity in utility levels into the unable subpopulation. The second line is the aggregate welfare of able individuals: Type II errors introduce horizontal inequity in utility levels at each net wage, in addition to changing individual welfare rankings 
across net wages. ${ }^{11}$ Finally, notice that $W^{N}(B, C ; \theta, 0,0)=W^{P}(B, C ; \theta)$.

The optimisation problem of the government is given by:

$$
\begin{array}{ll} 
& \max _{B, C} W^{N}\left(B, C ; \theta, p_{I}, p_{I I}\right) \\
\text { s.t. } & B+\left[\theta\left(1-p_{I}\right)+(1-\theta) p_{I I}\right] C=\beta, B \geq 0, C \geq 0
\end{array}
$$

Let us define (i) the aggregate smvi of categorical recipients and (ii) the aggregate smvi of those not receiving the categorical benefit (henceforth 'non-recipients') by:

$$
\begin{aligned}
\varphi_{R}^{N}\left(B, C ; \theta, p_{I}, p_{I I}\right) & \equiv \theta\left(1-p_{I}\right) u_{x}(B+C, 1)+(1-\theta) p_{I I} \int_{0}^{\infty} v(\omega, B+C) d F(\omega) \\
\varphi_{N R}^{N}\left(B ; \theta, p_{I}, p_{I I}\right) & \equiv \theta p_{I} u_{x}(B, 1)+(1-\theta)\left(1-p_{I I}\right) \int_{0}^{\infty} v_{M}(\omega, B) d F(\omega)
\end{aligned}
$$

with $\bar{\varphi}_{R}^{N}\left(B, C ; \theta, p_{I}, p_{I I}\right) \equiv \varphi_{R}^{N} /\left[\theta\left(1-p_{I}\right)+(1-\theta) p_{I I}\right]$ and $\bar{\varphi}_{N R}^{N}\left(B ; \theta, p_{I}, p_{I I}\right) \equiv \varphi_{N R}^{N} /\left[\theta p_{I}+\right.$ $\left.(1-\theta)\left(1-p_{I I}\right)\right]$ denoting the corresponding averages, respectively.

Letting $\hat{B}^{N}\left(\beta, \theta, p_{I}, p_{I I}\right)$ and $\hat{C}^{N}\left(\beta, \theta, p_{I}, p_{I I}\right)$ denote the optimal benefit levels, we have:

\section{Proposition 2a.}

(i) $\hat{C}^{N}\left(\beta, \theta, p_{I}, p_{I I}\right)\left\{\begin{array}{l}> \\ =\end{array}\right\} 0 \forall p_{I}+p_{I I}\left\{\begin{array}{c}< \\ =\end{array}\right\} 1$.

(ii) $\hat{B}^{N}\left(\beta, \theta, p_{I}, p_{I I}\right)=\beta \forall p_{I}+p_{I I}=1$. But when $p_{I}+p_{I I}<1$ the optimal benefits satisfy:

$$
\bar{\varphi}_{N R}^{N}\left(\hat{B}^{N} ; \theta, p_{I}, p_{I I}\right) \leq \bar{\varphi}_{R}^{N}\left(\hat{B}^{N}, \hat{C}^{N} ; \theta, p_{I}, p_{I I}\right) ; \hat{B}^{N} \geq 0
$$

where the pair of inequalities hold with complementary slackness. Furthermore, in this case if $p_{I}>0$ then certainly $\hat{B}^{N}\left(\beta, \theta, p_{I}, p_{I I}\right)>0$, but if $p_{I}=0$ then $\hat{B}^{N}\left(\beta, \theta, p_{I}, p_{I I}\right)>$ 0 only if $\beta>\bar{\beta}^{N}$, defined as the critical budget satisfying:

$$
\bar{\varphi}_{N R}^{N}\left(0 ; \theta, 0, p_{I I}\right) \equiv \bar{\varphi}_{R}^{N}\left(0, \frac{\bar{\beta}^{N}}{\theta+(1-\theta) p_{I I}} ; \theta, 0, p_{I I}\right)
$$

Proof: See supplementary online Appendix.

The principal messages from Proposition 2a are that (i) a necessary and sufficient condition to provide a categorical benefit is that the awards test has some discriminatory power; and

\footnotetext{
${ }^{11}$ For example, an individual with $\omega_{0}<\omega_{1}$ may have $v\left(\omega_{0}, B+C\right)>v\left(\omega_{1}, B\right)$.
} 
where this holds (ii) it is optimal to provide a universal benefit whenever Type I errors occur, or otherwise if the budget is sufficiently large. In general, in any optimum where both benefits are provided the average smvi of categorical recipients will equate with the average smvi of non-recipients, as stated in (14).

To provide the intuition for Proposition 2a(i) we compare welfare under an arbitrary budgetfeasible targeted system $(B \geq 0, C>0)$ with that under a pure universal system:

$$
\begin{aligned}
W^{N}-W^{U}= & \theta\left\{\begin{array}{c}
\left\langle\left(1-p_{I}\right) u(B+C, 1)+p_{I} u(B, 1)\right\rangle-u\left[B+\left(1-p_{I}\right) C, 1\right] \\
+\left\langle u\left[B+\left(1-p_{I}\right) C, 1\right]-u(\beta, 1)\right\rangle
\end{array}\right\} \\
& +(1-\theta) \int_{0}^{\infty}\left\{\begin{array}{c}
\left\langle p_{I I} v(\omega, B+C)+\left(1-p_{I I}\right) v(\omega, B)\right\rangle-v\left[\omega, B+p_{I I} C\right] \\
+\left\langle v\left[\omega, B+p_{I I} C\right]-v(\omega, \beta)\right\rangle
\end{array}\right\} d F(\omega)
\end{aligned}
$$

The two pairs of curly braces multiplied by $\theta$ and $(1-\theta)$ concern the unable and able subpopulations, respectively. Within both pairs of braces the first line is negative by the concavity of individual utility: the utility of consuming the average benefit - i.e. $B+\left(1-p_{I}\right) C$ for the unable and $B+p_{I I} C$ for the able - exceeds the expected utility. This captures the within-group effect of classification errors. Next, the second line is positive for the unable but negative for the able whenever $p_{I}+p_{I I}<1$ : this arises because the unable (able) receive a higher (lower) benefit on average than under a pure universal system. However, both second lines are zero if instead $p_{I}+p_{I I}=1$ because a targeted system provides no more on average to the unable than the able. Consequently, if $p_{I}+p_{I I}=1$ a targeted system does worse than a pure universal system within-group and no better between-group. This explains why $\hat{C}^{N}=0$ when $p_{I}+p_{I I}=1$.

The intuition for Proposition 2a(ii) is as follows: if Type I errors occur a universal benefit is necessary to avoid some unable individuals having no income; but otherwise a universal benefit should only be provided if the budget exceeds that which finances categorical transfers so as to equate the average smvi of categorical recipients with that of able non-recipients.

We now turn our attention to how classification errors affect maximum social welfare, as defined by $V^{N}\left(\beta, \theta, p_{I}, p_{I I}\right) \equiv W^{N}\left(\hat{B}^{N}, \hat{C}^{N} ; \theta, p_{I}, p_{I I}\right)$.

Proposition 2b. Maximum social welfare decreases with both error propensities:

$$
\frac{\partial V^{N}\left(\beta, \theta, p_{I}, p_{I I}\right)}{\partial p_{I}}<0, \frac{\partial V^{N}\left(\beta, \theta, p_{I}, p_{I I}\right)}{\partial p_{I I}}<0 \quad \forall p_{I}+p_{I I}<1
$$


Proof: See supplementary online Appendix.

In reality reforms to the awards technology may correspond to either (i) genuine improvements in discriminatory power through investment in technology which lowers $p_{I}, p_{I I}$ or both; or simply (ii) shifts in test bias - i.e. making the test more lenient/stringent (see Goodin, 1985). The second case implies a tradeoff between error types: a reduction in $p_{I}$ $\left(p_{I I}\right)$ is brought about through making the awards test more lenient (stringent), which in turn increases $p_{I I}\left(p_{I}\right)$. Proposition $2 \mathrm{~b}$ implies that there will be a marginal welfare gain to reforms of the first type. In reality these must be compared with the marginal investment costs. Meanwhile, the overall welfare effect of reforms of the second type will depend on the relative sizes of $\left|\partial V^{N} / \partial p_{I}\right|$ and $\left|\partial V^{N} / \partial p_{I I}\right|$, respectively.

\subsection{Imperfect Discrimination: Full Enforcement}

When the condition that recipients of $C$ do not work is fully enforced, we know from (5) that the only able individuals $\omega \leq \overline{\bar{\omega}}(B, C)$ will apply for $C$. Social welfare is therefore now:

$$
\begin{aligned}
& W^{F}\left(B, C ; \theta, p_{I}, p_{I I}\right) \\
= & \theta\left[\left(1-p_{I}\right) u(B+C, 1)+p_{I} u(B, 1)\right] \\
+ & (1-\theta)\left\{\begin{array}{c}
p_{I I} F(\overline{\bar{\omega}}) u(B+C, 1)+\left(1-p_{I I}\right)\left[F(\bar{\omega}) u(B, 1)+\int_{\bar{\omega}}^{\overline{\bar{\omega}}} v(\omega, B) d F(\omega)\right] \\
+\int_{\overline{\bar{\omega}}}^{\infty} v(\omega, B) d F(\omega)
\end{array}\right\}
\end{aligned}
$$

The first line on the right side of (18) is the aggregate welfare of unable individuals. The second line is the aggregate welfare of able individuals. Type II classification errors introduce horizontal inequity at each net wage $\omega \leq \overline{\bar{\omega}}$, where as (18) illustrates applicants are composed of both the voluntarily unemployed and those who work when receiving only $B$. The closer $\overline{\bar{\omega}}$ is to $\bar{\omega}$ the closer the average welfare of able applicants is to that of unable applicants.

The government budget constraint is given by:

$$
B+\left[\theta\left(1-p_{I}\right)+(1-\theta) F(\overline{\bar{\omega}}) p_{I I}\right] C \leq \beta
$$

To facilitate the intuition for the analysis which follows, let $C^{F}\left(B ; \beta, \theta, p_{I}, p_{I I}\right)$ be the level of categorical benefit that exhausts the budget constraint for any given $B \leq \beta$. Formally:

$$
\left\{\theta\left(1-p_{I}\right)+(1-\theta) F\left[\overline{\bar{\omega}}\left(B, C^{F}\right)\right] p_{I I}\right\} C^{F} \equiv \beta-B
$$


Differentiating (20) w.r.t. $B$ thus yields:

$$
\frac{\partial C^{F}}{\partial B}=-\frac{1+(1-\theta) f(\overline{\bar{\omega}}) p_{I I} \overline{\bar{\omega}}_{B} C^{F}}{\left[\theta\left(1-p_{I}\right)+(1-\theta) F(\overline{\bar{\omega}}) p_{I I}\right]+(1-\theta) f(\overline{\bar{\omega}}) p_{I I} \overline{\bar{\omega}}_{C} C^{F}}<0
$$

We henceforth assume that (i) $\partial C^{F} / \partial B<-1$, such that the total benefit income of categorical recipients falls with the universal benefit; and (ii) $\partial^{2} C^{F} / \partial B^{2} \leq 0$. Since $\overline{\bar{\omega}}_{B}<\overline{\bar{\omega}}_{C}$ :

$$
\frac{d \overline{\bar{\omega}}\left(B, C^{F}\right)}{d B}=\frac{\left[\theta\left(1-p_{I}\right)+(1-\theta) F(\overline{\bar{\omega}}) p_{I I}\right] \cdot \overline{\bar{\omega}}_{B}-\overline{\bar{\omega}}_{C}}{\left[\theta\left(1-p_{I}\right)+(1-\theta) F(\overline{\bar{\omega}}) p_{I I}\right]+(1-\theta) f(\overline{\bar{\omega}}) p_{I I} \overline{\bar{\omega}}_{C} C^{F}}<0
$$

A budget-balanced increase in the universal benefit thus reduces the number of able individuals who choose to apply for the categorical benefit.

The optimisation problem of the government is thus now given by:

$$
\begin{aligned}
& \quad \max _{B, C} W^{F}\left(B, C ; \theta, p_{I}, p_{I I}\right) \\
& \text { s.t } B+\left[\theta\left(1-p_{I}\right)+(1-\theta) F(\overline{\bar{\omega}}) p_{I I}\right] C=\beta ; \quad B \geq 0, C \geq 0
\end{aligned}
$$

One cannot in general guarantee that social welfare will be concave in budget-balanced changes in the benefit levels, or indeed that there will always be a unique solution to the above optimisation problem (see also Stern, 1982). We briefly discuss this below. Thereafter, the key results presented in this section are derived under the assumption that first-order conditions are sufficient to characterise a unique optima.

We define the aggregate smvi of categorical recipients and non-recipients, respectively, by:

$$
\begin{aligned}
& \varphi_{R}^{F}\left(B, C, \overline{\bar{\omega}} ; \theta, p_{I}, p_{I I}\right) \equiv\left[\theta\left(1-p_{I}\right)+(1-\theta) F(\overline{\bar{\omega}}) p_{I I}\right] u_{x}(B+C, 1) \\
& \varphi_{N R}^{F}\left(B, \overline{\bar{\omega}} ; \theta, p_{I}, p_{I I}\right) \equiv \theta p_{I} u_{x}(B, 1) \\
&+(1-\theta)\left\{\left(1-p_{I I}\right) \int_{0}^{\bar{\omega}} v_{M}(\omega, B) d F(\omega)+\int_{\overline{\bar{\omega}}}^{\infty} v_{M}(\omega, B) d F(\omega)\right\}
\end{aligned}
$$

Contrary the No Enforcement case, these functions may be non-monotonic in budget-balanced changes in the benefit levels. Substituting $C^{F}$ into (24) and differentiating w.r.t. $B$ yields: 


$$
\begin{aligned}
\frac{d \varphi_{R}^{F}\left(B, C^{F}, \overline{\bar{\omega}} ; \theta, p_{I}, p_{I I}\right)}{d B} & =\underbrace{\frac{\partial \varphi_{R}^{F}}{\partial B} \cdot\left(1+\frac{\partial C^{F}}{\partial B}\right)}_{>0}+\underbrace{\frac{\partial \varphi_{R}^{F}}{\partial \overline{\bar{\omega}}} \cdot \frac{d \overline{\bar{\omega}}\left(B, C^{F}\right)}{d B}}_{<0} \\
\frac{d \varphi_{N R}^{F}\left(B, \overline{\bar{\omega}} ; \theta, p_{I}, p_{I I}\right)}{d B} & =\underbrace{\frac{\partial \varphi_{N R}^{F}}{\partial B}}_{<0}+\underbrace{\frac{\partial \varphi_{N R}^{F}}{\partial \overline{\bar{\omega}}} \cdot \frac{d \overline{\bar{\omega}}\left(B, C^{F}\right)}{d B}}_{>0}
\end{aligned}
$$

A budget-balanced increase in the universal benefit thus generates an overall ambiguous effect on the aggregate smvi of categorical recipients $\left(\varphi_{R}^{F}\right)$ and non-recipients $\left(\varphi_{N R}^{F}\right)$, respectively. This arises precisely because changes in $\overline{\bar{\omega}}$ move the two components of either aggregate smvi (i.e. individual smvi, number of individuals) in opposite directions.

The above discussion highlights the additional complexity that endogenising application decisions to the benefit levels - via a fully enforced no-work condition - brings to the analysis. Just how social welfare changes with budget-balanced changes in spending will depend on undefined properties of both the density function (e.g. $\left.f^{\prime}(\omega)\right)$ and preferences (e.g. $\left.u_{x x x}\right)$.

Letting $\hat{B}^{F}\left(\beta, \theta, p_{I}, p_{I I}\right)$ and $\hat{C}^{F}\left(\beta, \theta, p_{I}, p_{I I}\right)$ denote the optimal benefit levels, we have:

\section{Proposition 3a.}

(i) $\hat{C}^{F}\left(\beta, \theta, p_{I}, p_{I I}\right)>0 \forall p_{I}+p_{I I} \leq 1$

(ii) The optimal benefits satisfy:

$$
\varphi_{N R}^{F}\left(\hat{B}^{F}, \overline{\bar{\omega}} ; \theta, p_{I}, p_{I I}\right) \leq \varphi_{R}^{F}\left(\hat{B}^{F}, \hat{C}^{F}, \overline{\bar{\omega}} ; \theta, p_{I}, p_{I I}\right) \cdot-\left[1+\frac{\partial C^{F}}{\partial B}\right] \quad ; \hat{B}^{F} \geq 0
$$

where the pair of inequalities hold with complementary slackness. An implication is that $\hat{B}^{F}\left(\beta, \theta, p_{I}, p_{I I}\right)>0$ if $p_{I}>0$.

Proof: See supplementary online Appendix.

The principal messages from Proposition 3a are that (i) it is optimal to provide a categorical benefit for all levels of discriminatory power, thus including the case of no discriminatory power; and (ii) it will be optimal to provide a universal benefit whenever Type I errors occur, and more generally as specified by (26). Indeed, in any optimum where both benefits are provided the aggregate smvi of non-recipients will equate with the aggregate smvi of categorical recipients, multiplied by the negative of the reduction in their total benefit income per unit increase in the universal benefit. 
To provide the intuition for Proposition 3a(i) consider a first-order Taylor approximation of $W^{F}-W^{U}$ around $\beta$ :

$$
\begin{aligned}
W^{F}-W^{U} & \approx \theta(1-\theta)\left[1-p_{I}-F(\overline{\bar{\omega}}) p_{I I}\right] C\left\{u_{x}(\beta, 1)-\int_{0}^{\infty} v_{M}(\omega, \beta) d F(\omega)\right\} \\
& +(1-\theta) p_{I I} F(\overline{\bar{\omega}}) C\left\{\frac{F[\bar{\omega}(\beta)]}{F(\overline{\bar{\omega}})} u_{x}(\beta, 1)-\int_{0}^{\infty} v_{M}(\omega, \beta) d F(\omega)\right\}
\end{aligned}
$$

This first line is positive at all levels of discriminatory power because $F(\overline{\bar{\omega}})<1$ for a finite benefit budget. ${ }^{12}$ This captures the fact that an arbitrary targeted system will, on average, award the unable with more benefit income than the able at all levels of discriminatory power. Next, the second line is likely to be positive for a small categorical benefit where $\overline{\bar{\omega}} \sim \bar{\omega}(\beta) \Rightarrow$ $F(\bar{\omega}) \sim F(\overline{\bar{\omega}})$. This line captures the fact that Type II errors actually redistribute within the able subpopulation because only those with $\omega \leq \overline{\bar{\omega}}$ will apply for the benefit. Indeed, the closer $F(\bar{\omega}) / F(\overline{\bar{\omega}})$ is to unity the larger this effect is, precisely because able individuals with $\omega<\bar{\omega}$ are formally equivalent to the unable from the perspective of smvi.

The intuition for Proposition 3a(ii) parallels its No Enforcement counterpart (i.e. Proposition $2 \mathrm{a}(\mathrm{ii})$ ). If Type I errors are made it is always optimal to provide a universal benefit to ensure rejected able individuals have some source of income to consume.

We now turn to discuss how classification errors affect maximum social welfare, as defined by the value function $V^{F}\left(\beta, \theta, p_{I}, p_{I I}\right) \equiv W^{F}\left(\hat{B}^{F}, \hat{C}^{F} ; \theta, p_{I}, p_{I I}\right)$.

Proposition 3b. Maximum social welfare is decreasing in the propensity to make Type I errors, but can be increasing in the propensity to make Type II errors:

$$
\begin{aligned}
& \frac{\partial V^{F}\left(\beta, \theta, p_{I}, p_{I I}\right)}{\partial p_{I}}<0 \quad \forall p_{I}+p_{I I} \leq 1 \\
& \frac{\partial V^{F}\left(\beta, \theta, p_{I}, p_{I I}\right)}{\partial p_{I I}}\left\{\begin{array}{l}
> \\
= \\
<
\end{array}\right\} 0 \forall p_{I}+p_{I I} \leq 1 \text { satisfying } \\
& {\left[u\left(\hat{B}^{F}+\hat{C}^{F}, 1\right)-\frac{1}{F(\overline{\bar{\omega}})} \int_{0}^{\bar{\omega}} v\left(\omega, \hat{B}^{F}\right) d F(\omega)\right]\left\{\begin{array}{l}
> \\
= \\
<
\end{array}\right\} \hat{\lambda}^{F} \hat{C}^{F} }
\end{aligned}
$$

A sufficient condition for welfare to be increasing in the Type II error propensity is $E>0 \Rightarrow$

\footnotetext{
${ }^{12}$ This follows from our assumption that $f(\omega)>0 \forall \omega \geq 0$.
} 
$\partial V^{F} / \partial p_{I I}>0$; where:

$$
E \equiv \frac{F\left(\bar{\omega}\left(\hat{B}^{F}\right)\right)}{\frac{\theta\left(1-p_{I}\right)}{(1-\theta) p_{I I}}+F(\overline{\bar{\omega}})} \cdot \underbrace{\frac{\overline{\bar{\omega}} f(\overline{\bar{\omega}})}{F(\overline{\bar{\omega}})}}_{\text {elasticity of } F \text { wrt } \overline{\bar{\omega}}} \cdot \underbrace{\frac{\hat{C}^{F} \overline{\bar{\omega}}_{C}}{\overline{\bar{\omega}}}}_{\text {elasticity of } \overline{\bar{\omega}} \operatorname{wrt} C}-\left[1-\frac{F\left(\bar{\omega}\left(\hat{B}^{F}\right)\right)}{F(\overline{\bar{\omega}})}\right]
$$

Proof: See supplementary online Appendix.

The intuition for Proposition 3b is as follows. Maximum social welfare may increase with the Type II error propensity because 'leakage' of categorical transfers to lower productivity able individuals plays a redistributive role within the able subpopulation. It is precisely because the fully enforced no-work condition restricts applications from able individuals to those of lower productivity that we attain this result. We can make a number of observations from (29). First, the closer $F(\bar{\omega}) / F(\overline{\bar{\omega}})$ is to unity - and thus the larger the fraction of able applicants with the same smvi as unable applicants - the more likely that $E>0$. Second, the first term in (29) implies that $E$ may increase with $p_{I}$ and $p_{I I}$, but decrease with $\theta$.

\subsection{A Numerical Example where $\partial V^{F} / \partial p_{I I}>0$}

Following Proposition 3b, we now provide a numerical example where $\partial V^{F} / \partial p_{I I}>0$. Let preferences take the Cobb-Douglas form $u(x, l)=x^{\alpha} l^{1-\alpha}$; where $\alpha=0.6$. Individual productivity, $n$, is exponentially distributed with rate parameter $\mu$. Given a tax rate $\tau$, this implies a net wage distribution $f(\omega)=[\mu /(1-\tau)] \exp [-\omega \mu /(1-\tau)]$. We set $\mu=3$ and $\tau=0.3$. Given our choices of $\alpha, \tau$ and $\mu$ the average wage individual ( $\omega=0.233)$ works roughly $3 / 5$ of their time endowment. Finally, we set $\theta=0.05$ and $\beta=0.05$, the latter of which is less than tax revenue under a pure universal system.

Figure 1 displays the numerical results. There are four subplots: within each (i) $p_{I I}$ is varied on the horizontal axis; whilst (ii) different curves are drawn for different values of $p_{I}$. Subplot (a) displays maximum welfare under Full Enforcement $\left(V^{F}\right)$ relative to that under Perfect Discrimination $\left(V^{P}\right)$. Subplot (b) displays the fraction of able applicants who are voluntarily unemployed at the optimum, i.e. $F\left[\bar{\omega}\left(\hat{B}^{F}\right)\right] / F\left[\overline{\bar{\omega}}\left(\hat{B}^{F}, \hat{C}^{F}\right]\right.$. Subplots (c) and (d) display the optimal benefits, $\hat{B}^{F}$ and $\hat{C}^{F}$, respectively.

In subplot (a) $V^{F}$ increases with $p_{I I}$ for $p_{I I}$ high enough. Further, $V^{F}$ can exceed $V^{P}$ (i.e. $V^{F} / V^{P}>1$ ) provided $p_{I}$ is not too high, thus capturing the redistributive role that 'leakage' of the categorical benefit plays within the able subpopulation. In subplot (b) the fraction of able applicants who are voluntarily unemployed - and so have the same smvi as unable 
Figure 1: Numerical Results with Cobb-Douglas preferences.

\begin{tabular}{|c|cc|cc|lc|}
\hline$-p_{I}=0$ & $\cdots$ & $p_{I}=0.2$ & $\cdots$ & $p_{I}=0.4$ & $\longmapsto$ & $p_{I}=0.6$ \\
\hline
\end{tabular}
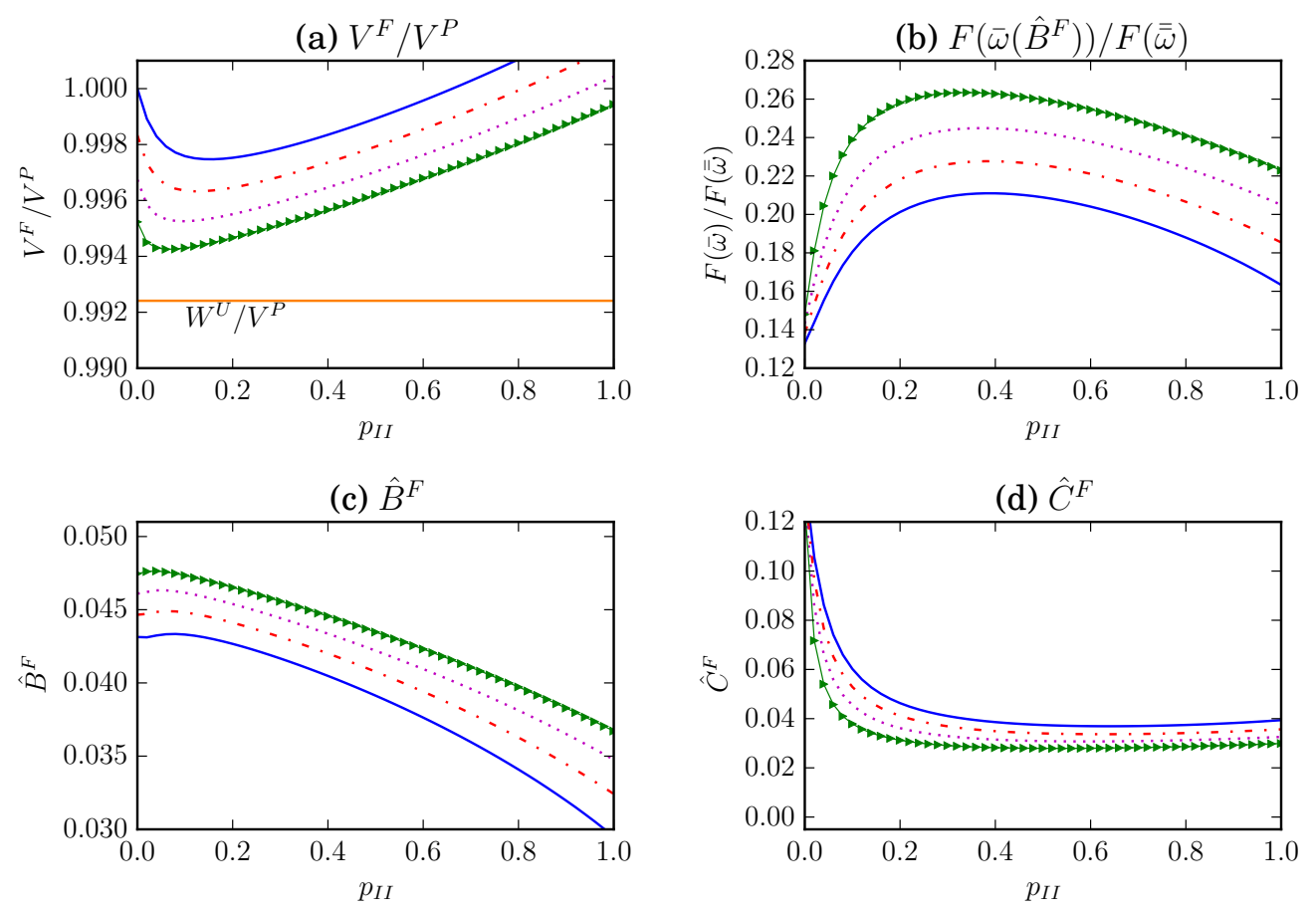

Notes. The line $W^{U} / V^{P}$ provides a lower welfare bound because the outcome $W^{U}$ is always feasible.

applicants - increases with $p_{I I}$ at lower values of $p_{I I}$. Finally, in subplots (c) and (d) the optimal benefits increase with $p_{I}$, but tend to fall with $p_{I I}$.

\subsection{Welfare Comparison: No Enforcement vs. Full Enforcement}

We now discuss how social welfare compares across the two enforcement regimes. Clearly, if $p_{I I}=0$ we have $V^{F}=V^{N}$; whilst if $p_{I}+p_{I I}=1$ then $V^{F}>V^{N}=W^{U}$ by Propositions 2a and $3 \mathrm{a}$, respectively. We thus focus on the intermediate cases $p_{I}+p_{I I}<1$.

Let $C^{N}\left(B ; \beta, \theta, p_{I}, p_{I I}\right) \equiv(\beta-B) /\left[\theta\left(1-p_{I}\right)+(1-\theta) p_{I I}\right]$ be the value of $C$ that exhausts the No Enforcement budget constraint for any given $B$ (and exogenous parameters). For a finite budget $\beta$ we thus have $C^{N}<C^{F} \forall p_{I}+p_{I I} \leq 1$; where $C^{F}$ is as defined in (20). For a given $B$ (and $\left.\beta, \theta, p_{I}, p_{I I}\right)$ there will thus be a critical wage $\tilde{\omega}(B)$ satisfying:

$$
v\left[\tilde{\omega}(B), B+C^{N}\right] \equiv u\left(B+C^{F}, 1\right)
$$


At $\omega=\tilde{\omega}$ an individual is indifferent between (i) receiving $C^{N}<C^{F}$ but being (effectively) unrestricted in their labour supply; and (ii) receiving $C^{F}>C^{N}$ but facing a fully enforced no-work condition. This no-work condition binds because $\tilde{\omega}(B)>\bar{\omega}\left(B+C^{F}\right)$.

Now suppose we compare maximum welfare under No Enforcement with welfare under an arbitrary Full Enforcement system where $B=\hat{B}^{N}$ and $C=C^{F}\left(\hat{B}^{N} ; \beta, \theta, p_{I}, p_{I I}\right)^{13}$ :

$$
\begin{aligned}
& W^{F}\left(\hat{B}^{N}, C^{F} ; \theta, p_{I}, p_{I I}\right)-V^{N}\left(\beta, \theta, p_{I}, p_{I I}\right) \\
&= \theta\left(1-p_{I}\right)\left[u\left(\hat{B}^{N}+C^{F}, 1\right)-u\left(\hat{B}^{N}+\hat{C}^{N}, 1\right)\right] \\
&+(1-\theta) p_{I I}\left\{\begin{array}{c}
\int_{0}^{\tilde{\omega}\left(\hat{B}^{N}\right)}\left[u\left(\hat{B}^{N}+C^{F}, 1\right)-v\left(\omega, \hat{B}^{N}+\hat{C}^{N}\right)\right] d F(\omega) \\
+\int_{\tilde{\omega}\left(\bar{B}^{N}\right)}^{\bar{\omega}\left(\bar{B}^{F}\right)}\left[u\left(\hat{B}^{N}+C^{F}, 1\right)-v\left(\omega, \hat{B}^{N}+\hat{C}^{N}\right)\right] d F(\omega) \\
+\int_{\overline{\bar{\omega}}\left(\bar{B}, C^{F}\right)}^{\infty}\left[v\left(\omega, \hat{B}^{N}\right)-v\left(\omega, \bar{B}+C^{N}\right)\right] d F(\omega)
\end{array}\right\}
\end{aligned}
$$

The first line concerns unable categorical recipients: it is positive because $C^{F}>C^{N}$. The second line concerns able individuals and is composed of the three subcases within curly braces. First, categorical recipients with $\omega \in(0, \tilde{\omega})$ are better-off under Full Enforcement than under No Enforcement. Within this subgroup, the fully enforced no-work condition binds for those with $\omega \in\left(\bar{\omega}\left(\hat{B}^{N}+C^{F}\right), \tilde{\omega}\right]$, but these individuals would not be willing to forgo $C^{F}-C^{N}$ in exchange for a full relaxation of the enforcement of this constraint. Second, however, categorical recipients with $\omega \in(\tilde{\omega}, \overline{\bar{\omega}}]$ would be willing to make this exchange and so are worse-off under Full Enforcement. Third and finally, individuals with $\omega \in(\overline{\bar{\omega}}, \infty)$ do not apply for the categorical benefit and so are by definition worse-off under Full Enforcement than their counterparts who are awarded $C^{N}$ under the No Enforcement regime.

If the redistributive gains that the arbitrary Full Enforcement system provides to both unable individuals and lower productivity individuals with $\omega \in(0, \tilde{\omega})$ offsets any losses further up the productivity distribution, it will certainly be the case that welfare under an optimally chosen Full Enforcement system will exceed maximum welfare under No Enforcement.

\section{Extension: Analysis Within Optimal Tax Framework}

This section extends the analysis to the more general case where benefit expenditure is financed through a linear income tax, which is now a choice variable. A productivity $n$ individual has net wage $\omega(n, \tau) \equiv n(1-\tau)$. Let $f(n)$ and $F(n)$ be the productivity density

\footnotetext{
${ }^{13}$ The universal benefit is thus constant across enforcement regimes.
} 
and distribution functions, respectively: $f$ satisfies the assumptions made in Section 2.2. We denote individual gross earnings by $y(n, 1-\tau, M) \equiv n H^{*}[\omega(n, \tau), M]$ and average gross earnings across able individuals by $\bar{y}(n, 1-\tau, M)=\int_{0}^{\infty} y(n, 1-\tau, M) d F(n)$.

The net smvi of a productivity $n$ individual is (Viard, 2001a,b):

$$
s(n, \tau, M, \lambda)= \begin{cases}u_{x}(M, 1) & : n \leq \bar{n}(\tau, M) \\ v_{M}[\omega(n, \tau), M]+\lambda \tau y_{M}(n, \tau, M) & : n>\bar{n}(\tau, M)\end{cases}
$$

where $\bar{n}(\tau, M)=\bar{\omega}(M) /(1-\tau)$ and $\lambda$ is the Lagrange multiplier for the government budget constraint. The function $s$ is the individual smvi net of, in welfare units, any reduction in taxes paid via the labour response to unearned income. We assume that $\partial s / \partial M=$ $v_{M M}+\lambda \tau y_{M M}<0$, which simply requires that $y_{M M}=n H_{M M}^{*}$ be sufficiently small.

We now proceed to highlight the key results. To illustrate that the results are analogous to the fixed budget case we use the same proposition numbers followed by a prime.

\subsection{Perfect Discrimination}

The perfect discrimination problem is now described by:

$$
\begin{array}{ll} 
& \max _{\tau, B, C} W^{P}(\tau, B, C ; \theta) \\
\text { s.t. } & B+\theta C=(1-\theta) \tau \bar{y}, \tau \in(0,1) ; B \geq 0 ; C \geq 0 .
\end{array}
$$

where $W^{P}$ is as defined in (7), written in terms of $n$ and $\tau$. Solving this problem yields:

\section{Proposition $\mathbf{1}^{\prime}$}

(i) $\hat{C}^{P}(\theta)>0$

(ii) The optimal benefits satisfy:

$$
\int_{0}^{\infty} s\left(n, \hat{\tau}^{P}, \hat{B}^{P}, \hat{\lambda}^{P}\right) d F(n) \leq u_{x}\left(\hat{B}^{P}+\hat{C}^{P}, 1\right) \equiv \hat{\lambda}^{P} ; \hat{B}^{P} \geq 0
$$

where the pair of inequalities hold with complementary slackness.

(iii) For $\kappa=\hat{\lambda}^{P}-\int_{0}^{\infty} s d F(n)$ and $r=y / \bar{y}$ : 


$$
\frac{\hat{\tau}^{P}}{1-\hat{\tau}^{P}}= \begin{cases}\frac{\kappa-\operatorname{Cov}(r, s)}{\hat{\lambda}^{P} \int_{0}^{\infty}(r \cdot e) d F(n)} & : \kappa>0 \\ \frac{-\operatorname{Cov}(y, s)}{\hat{\lambda}^{P} \int_{0}^{\infty}(y \cdot e) d F(n)} & : \kappa=0\end{cases}
$$

and $e \equiv(1-\tau) y^{-1} \partial y^{c} / \partial(1-\tau)$ is the elasticity of compensated earnings, $y^{c}$, w.r.t. $1-\tau$.

\section{Proof: See supplementary online Appendix.}

Proposition $1^{\prime}$ states that (i) it is optimal to provide a categorical benefit; but (ii) a universal benefit will only be provided if it is optimal to generate tax revenue in excess of that which finances categorical transfers to the point that eliminates inequality in the average net smvi across the unable and able subpopulations. The implicit expression characterising the optimal tax rate captures the equity (numerator) - efficiency (denominator) tradeoff inherent in income taxation (see Slack, 2015). Whenever $\kappa>0$ there are two equity issues: inequality in the average net smvi between the unable and able subpopulations; and inequality in the individual net smvi within the able subpopulation. The former is captured by $\kappa$, whilst the latter is captured by the negative of the covariance between relative gross earnings and the net smvi. ${ }^{14}$ The denominator illustrates that, ceteris paribus, higher compensated earnings elasticities imply lower tax rates, with weight placed on those with high productivities and productivities at which the population is most dense. Finally, if $\kappa=0$ the optimal tax expression reduces to the standard form in the literature (Atkinson and Stiglitz, 1980).

Simple example where $\kappa>0$. Let $u=\log \left[x-H^{1+\epsilon} /(1+\epsilon)\right]$, where $1 / \epsilon$ is the constant labour elasticity (see Atkinson, 1990). Consider the problem:

$$
\max _{\tau} W^{P}(\tau, 0, C(\tau) ; \theta) \text { s.t. } C(\tau)=\left(\frac{1-\theta}{\theta}\right) \tau \int_{0}^{\infty} y(n, 1-\tau) d F(n)
$$

Let (i) $\hat{\tau}_{C}$ be the solution to $(36)$ and (ii) $\tau_{\kappa}$ be the level of taxation that finances categorical transfers so as to eliminate between-group inequality (i.e. set $\kappa=0$ ). Formally:

$$
\hat{\tau}_{C}(\theta)=\theta\left(\frac{\epsilon}{1+\epsilon}\right), \tau_{\kappa}(\theta)=\frac{\theta\left(\frac{\epsilon}{1+\epsilon}\right)}{(1-\theta) \int_{0}^{\infty} n^{\frac{1+\epsilon}{\epsilon}} d F(n) \cdot \int_{0}^{\infty} n^{-\frac{1+\epsilon}{\epsilon}} d F(n)+\theta\left(\frac{\epsilon}{1+\epsilon}\right)}
$$

\footnotetext{
${ }^{14}$ Provided $\partial y / \partial n>0$ and $\partial s / \partial n<0$ this covariance will be negative, capturing a desire to redistribute from those of low net smvi (high productivity) to those of high net smvi (lower productivity).
} 
where $\partial \tau_{\kappa} / \partial \theta>0$. Since $\tau_{\kappa}(0)=\hat{\tau}_{C}(0)$ whilst $\tau_{\kappa}(1)>\hat{\tau}_{C}(1)$ there must be some critical value of $\theta \in(0,1), \tilde{\theta}$, satisfying $\hat{\tau}_{C}(\tilde{\theta})=\tau_{k}(\tilde{\theta})$ and $\hat{\tau}_{C}<\tau_{k} \forall \theta>\tilde{\theta}$ (see Slack, 2016).

\subsection{Imperfect Discrimination: No Enforcement}

The No Enforcement problem is now described by:

$$
\begin{aligned}
& \max _{\tau, B, C} W^{N}\left(\tau, B, C ; \theta, p_{I}, p_{I I}\right) \\
\text { s.t. } & B+\left[\theta\left(1-p_{I}\right)+(1-\theta) p_{I I}\right] C \\
= & (1-\theta) \tau\left[p_{I I} \bar{y}(1-\tau, B+C)+\left(1-p_{I I}\right) \bar{y}(1-\tau, B)\right], \tau \in(0,1) ; B \geq 0 ; C \geq 0 .
\end{aligned}
$$

where $W^{N}$ is as defined in (11), written in terms of $n$ and $\tau$. As in (13) from the fixed budget analysis, we define the aggregate smvi of categorical recipients and non-recipients by:

$$
\begin{aligned}
\varphi_{R}^{N}\left(\tau, B, C, \lambda ; \theta, p_{I}, p_{I I}\right) & \equiv \theta\left(1-p_{I}\right) u_{x}(B+C, 1)+(1-\theta) p_{I I} \int_{0}^{\infty} s(n, \tau, B+C, \lambda) d F(n) \\
\varphi_{N R}^{N}\left(\tau, B, \lambda ; \theta, p_{I}, p_{I I}\right) & \equiv \theta p_{I} u_{x}(B, 1)+(1-\theta) p_{I I} \int_{0}^{\infty} s(n, \tau, B, \lambda) d F(n)
\end{aligned}
$$

with averages $\bar{\varphi}_{R}^{N} \equiv \varphi_{R}^{N} /\left[\theta\left(1-p_{I}\right)+(1-\theta) p_{I I}\right]$ and $\bar{\varphi}_{N R}^{N} \equiv \varphi_{N R}^{N} /\left[\theta p_{I}+(1-\theta)\left(1-p_{I I}\right)\right.$.

Letting $\hat{\tau}^{N}\left(\theta, p_{I}, p_{I I}\right), \hat{B}^{N}\left(\theta, p_{I}, p_{I I}\right)$ and $\hat{C}^{N}\left(\theta, p_{I}, p_{I I}\right)$ be the solutions to (38), we have:

\section{Proposition $2 \mathrm{a}^{\prime}$}

(i) $\hat{C}^{N}\left(\theta, p_{I}, p_{I I}\right)\left\{\begin{array}{l}> \\ =\end{array}\right\} 0 \forall p_{I}+p_{I I}\left\{\begin{array}{l}< \\ =\end{array}\right\} 1$

(ii) When $p_{I}+p_{I I}<1$ the optimal benefits satisfy:

$$
\bar{\varphi}_{N R}^{N}\left(\hat{\tau}^{N}, \hat{B}^{N}, \hat{\lambda}^{N} ; \theta, p_{I}, p_{I I}\right) \leq \bar{\varphi}_{R}^{N}\left(\hat{\tau}^{N}, \hat{B}^{N}, \hat{C}^{N}, \hat{\lambda}^{N} ; \theta, p_{I}, p_{I I}\right) ; \hat{B}^{N} \geq 0
$$

where the pair of inequalities hold with complementary slackness. An implication is that $\hat{B}^{N}\left(\theta, p_{I}, p_{I I}\right)>0$ if $p_{I}>0$.

(iii) The optimal tax rate is implicitly characterised by:

$$
\frac{\hat{\tau}^{N}}{1-\hat{\tau}^{N}}=\frac{\int_{0}^{\infty}\left[\left.p_{I I} y\right|_{\hat{B}^{N}+\hat{C}^{N}}\left(\hat{\lambda}^{N}-\left.s\right|_{\hat{B}^{N}+\hat{C}^{N}}\right)+\left.\left(1-p_{I I}\right) y\right|_{\hat{B}^{N}}\left(\hat{\lambda}^{N}-\left.s\right|_{\hat{B}^{N}}\right)\right] d F(n)}{\hat{\lambda}^{N} \int_{0}^{\infty}\left[\left.p_{I I}(y \cdot e)\right|_{\hat{B}^{N}+\hat{C}^{N}}+\left.\left(1-p_{I I}\right)(y \cdot e)\right|_{\hat{B}^{N}}\right] d F(n)}
$$


where to save on notation $\left.y\right|_{M}=y(n, 1-\tau, M)$ and $\left.s\right|_{M}=s(n, \tau, M, \lambda)$.

Proof: See supplementary online Appendix.

Analogous to the fixed budget analysis, Proposition 2a' states that (i) it is optimal to provide a categorical benefit only if the awards test has positive discriminatory power; whilst (ii) it is optimal to provide a universal benefit whenever Type I errors are made or else whenever the average net smvi of non-recipients exceeds that of recipients when evaluated at $B=0$. The optimal tax expression in (40) has equity considerations in the numerator and efficiency considerations in the denominator. Loosely speaking, it resembles a weighted average of the standard optimal linear income tax expression, capturing the fact that at any given productivity some individuals receive $B$ in benefit income whilst others receive $B+C$.

Letting $V^{N}\left(\theta, p_{I}, p_{I I}\right) \equiv W^{N}\left(\hat{\tau}^{N}, \hat{B}^{N}, \hat{C}^{N} ; \theta, p_{I}, p_{I I}\right)$ we now state the following result.

Proposition $\mathbf{2} \mathbf{b}^{\prime}$. Maximum social welfare is decreasing in both error propensities:

$$
\frac{\partial V^{N}\left(\theta, p_{I}, p_{I I}\right)}{\partial p_{I}}<0, \frac{\partial V^{N}\left(\theta, p_{I}, p_{I I}\right)}{\partial p_{I I}}<0 \quad \forall p_{I}+p_{I I}<1
$$

Proof: See supplementary online Appendix.

\subsection{Imperfect Discrimination: Full Enforcement}

The Full Enforcement optimisation problem is now described by:

$$
\begin{array}{ll} 
& \max _{\tau, B, C} W^{F}\left(\tau, B, C ; \theta, p_{I}, p_{I I}\right) \\
\text { s.t. } & B+\left[\theta\left(1-p_{I}\right)+(1-\theta) F(\overline{\bar{n}}) p_{I I}\right] C \\
= & (1-\theta) \tau\left[\bar{y}(1-\tau, B)-p_{I I} \int_{0}^{\bar{n}} y(n, 1-\tau, B) d F(n)\right], \tau \in(0,1) ; B \geq 0 ; C \geq 0 .
\end{array}
$$

where $W^{F}$ is as defined in (18) (written in terms on $n$ and $\tau$ ) and intuitively $\overline{\bar{n}}=\overline{\bar{\omega}} /(1-\tau)$. As discussed in the fixed budget analysis, the endogeneity of applications decisions to the benefit levels means that social welfare may not be concave in budget-balanced changes in benefit levels. Further, (42) illustrates that the problem becomes much richer (and more complex) in an optimal tax setting because the choice of tax rate will affect the number of individuals who choose to apply for the categorical benefit, which in turn will affect both the benefit expenditure and tax revenue sides of the budget constraint. In all that follows we 
assume there to be a unique and well-defined solution to (42).

Let the aggregate net smvi of categorical recipients and non-recipients be, resectively:

$$
\begin{aligned}
\varphi_{R}^{F}\left(\tau, B, C, \overline{\bar{n}} ; \theta, p_{I}, p_{I I}\right) & =\left[\theta\left(1-p_{I}\right)+(1-\theta) F(\overline{\bar{n}}) p_{I I}\right] u_{x}(B+C, 1) \\
\varphi_{N R}^{F}\left(\tau, B, \lambda, \overline{\bar{n}} ; \theta, p_{I}, p_{I I}\right) & =\theta p_{I} u_{x}(B, 1) \\
& +(1-\theta)\left\{\int_{0}^{\infty} s(n, \tau, B, \lambda) d F(n)-p_{I I} \int_{0}^{\overline{\bar{n}}} s(n, \tau, B, \lambda) d F(n)\right\}
\end{aligned}
$$

The corresponding averages are $\bar{\varphi}_{R}^{F}=\varphi_{R}^{F} /\left[\theta\left(1-p_{I}\right)+(1-\theta) F(\overline{\bar{n}}) p_{I I}\right]$ and $\bar{\varphi}_{N R}^{F}=\varphi_{N R}^{F} /\left[\theta p_{I}+\right.$ $\left.(1-\theta)\left(1-F(\overline{\bar{n}}) p_{I I}\right)\right]$.

Letting $\hat{\tau}^{F}\left(\theta, p_{I}, p_{I I}\right), \hat{B}^{F}\left(\theta, p_{I}, p_{I I}\right)$ and $\hat{C}^{F}\left(\theta, p_{I}, p_{I I}\right)$ be the solutions to (42), we have:

\section{Proposition $3 \mathbf{a}^{\prime}$}

(i) $\hat{C}^{F}\left(\theta, p_{I}, p_{I I}\right)>0 \forall p_{I}+p_{I I} \leq 1$

(ii) The optimal benefits satisfy:

$$
\begin{aligned}
& \bar{\varphi}_{N R}^{F}\left(\hat{\tau}^{F}, \hat{B}^{F}, \hat{\lambda}^{F}, \overline{\bar{n}} ; \theta, p_{I}, p_{I I}\right) \\
\leq & \bar{\varphi}_{R}^{F}\left(\hat{B}^{F}, \hat{C}^{F}\right) \cdot\left\{\frac{1+\frac{(1-\theta) f(\overline{\bar{n}}) p_{I I}\left[\overline{\bar{n}}_{B}-\overline{\bar{n}}_{C}\right]\left[\hat{C}^{F}+\hat{\tau}^{F} y\left(\overline{\bar{n}}, 1-\hat{\tau}^{F}, \hat{B}^{F}\right)\right]}{\left[\theta p_{I}+(1-\theta)\left(1-F(\overline{\bar{n}}) p_{I I}\right)\right]}}{1+\frac{(1-\theta) f(\overline{\bar{n}}) p_{I I} \overline{\bar{n}}_{C}\left[\hat{C}^{F}+\hat{\tau}^{F} y\left(\overline{\bar{n}}, 1-\hat{\tau}^{F}, \hat{B}^{F}\right)\right]}{\left[\theta\left(1-p_{I}\right)+(1-\theta) F(\overline{\bar{n}}) p_{I I}\right]}}\right\} ; \hat{B}^{F} \geq 0
\end{aligned}
$$

where the pair of inequalities hold with complementary slackness. An implication is that $\hat{B}^{F}\left(\theta, p_{I}, p_{I I}\right)$ if $p_{I}>0$.

(iii) The optimal tax rate is implicitly characterised by:

$$
\frac{\hat{\tau}^{F}}{1-\hat{\tau}^{F}}=\frac{\left[\left.\int_{0}^{\infty} y\right|_{\hat{B}^{F}}\left(\hat{\lambda}^{F}-\left.s\right|_{\hat{B}^{F}}\right) d F(n)-\left.p_{I I} \int_{0}^{\overline{\bar{n}}} y\right|_{\hat{B}^{F}}\left(\hat{\lambda}^{F}-\left.s\right|_{\hat{B}^{F}}\right) d F(n)\right]-p_{I I} \hat{\lambda}^{F} f(\overline{\bar{n}}) \frac{\partial \overline{\bar{n}}}{\partial \tau} \hat{C}^{F}}{\hat{\lambda}^{F}\left\{\left[\left.\int_{0}^{\infty}(y \cdot e)\right|_{\hat{B}^{F}} d F(n)-\left.p_{I I} \int_{0}^{\overline{\bar{n}}}(y \cdot e)\right|_{\hat{B}^{F}} d F(n)\right]-p_{I I} F(\overline{\bar{n}}) \phi \eta y\left(\overline{\bar{n}}, 1-\hat{\tau}^{F}, \hat{B}^{F}\right)\right\}}
$$

where to save on notation $\left.y\right|_{M}=y(n, 1-\tau, M)$ and $\left.s\right|_{M}=s(n, \tau, M, \lambda) ; \phi \equiv \overline{\bar{n}} f(\overline{\bar{n}}) / F(\overline{\bar{n}})$ and $\eta \equiv-(1-\tau)(\overline{\bar{n}})^{-1} \partial \overline{\bar{n}} / \partial(1-\tau)$.

Proof: See supplementary online Appendix.

Analogous to the fixed budget analysis, Proposition $3 \mathrm{a}^{\prime}$ states that (i) it is optimal to provide a categorical benefit at all levels of discriminatory power; whilst (ii) the conditions under 
which a universal benefit will be provided are given by (43). The left side of (43) is the average net smvi of non-recipients, whilst the right side is the average smvi of categorical recipients multiplied by the negative of change in their total benefit income per unit increase in the universal benefit, abstracting from labour responses to unearned income. ${ }^{15}$

The optimal tax expression in (44) is more complex than that under No Enforcement due to the effect of the tax rate on application decisions. We first discuss the equity considerations in the numerator. The terms within square brackets capture the aggregate difference between the shadow price and individual net smvi, weighted by gross earnings. Notice that this aggregate is net of able applicants who are awarded the categorical benefit and thus who do not work. Ceteris paribus, if $s>\hat{\lambda}^{F}$ for individuals with $n \in[0, \overline{\bar{n}}]$ an increase in $p_{I I}$ may act to increase the tax rate because individuals who the government would not wish to tax highly - for the purpose of financing categorical transfers to the unable - are taken out of the tax system. Meanwhile, the second term captures the fact that a ceteris paribus increase in the tax rate increases the number of able individuals who apply for the categorical benefit. To the extent that this redistributes within the able subpopulation, this may act to lower the tax rate because there is less need for universal benefit expenditure. ${ }^{16}$

We now discuss the efficiency considerations in the denominator. The terms in square braces capture the aggregate responsiveness of compensated gross earnings to the net-of-tax rate, whilst the final term captures the fact that an increase in the tax rate induces more able individuals to apply for the categorical benefit, in turn resulting in a loss of tax revenue in the extensive margin. Whilst Type II errors may act to lessen the first concern (because fewer individuals respond to the tax rate), they may increase the latter (because more individuals stop working in response to an increase in the tax rate).

Letting $V^{F}\left(\theta, p_{I}, p_{I I}\right) \equiv W^{F}\left(\hat{\tau}^{N}, \hat{B}^{N}, \hat{C}^{N} ; \theta, p_{I}, p_{I I}\right)$ we now state the following result.

Proposition $3 \mathbf{b}^{\prime}$. Maximum social welfare is decreasing in the propensity to make Type I errors, but may be increasing or decreasing in the propensity to make Type II errors:

$$
\begin{aligned}
& \frac{\partial V^{F}\left(\theta, p_{I}, p_{I I}\right)}{\partial p_{I}}<0 \forall p_{I}+p_{I I} \leq 1 \\
& \left.\frac{\partial V^{F}\left(\theta, p_{I}, p_{I I}\right)}{\partial p_{I I}}\left\{\begin{array}{l}
> \\
= \\
<
\end{array}\right\} 0 \Leftrightarrow \frac{u\left(\hat{B}^{F}+\hat{C}^{F}, 1\right)-\frac{1}{F(\overline{\bar{n}})} \int_{0}^{\overline{\bar{n}}} v\left[\omega\left(n, \hat{\tau}^{F}\right), \hat{B}^{F}\right] d F(n)}{\hat{\lambda}^{F}\left[\hat{C}^{F}+\hat{\tau}^{F} \frac{1}{F(\overline{\bar{n}})} \int_{0}^{\bar{n}} y\left(n, 1-\hat{\tau}^{F}, \hat{B}^{F}\right) d F(n)\right]} \begin{array}{l}
> \\
= \\
<
\end{array}\right\}
\end{aligned}
$$

\footnotetext{
${ }^{15}$ The income effects attached to an increase in $B$ are captured through $s$ in the left side of (43). This is why we cannot write the terms in curly braces on the right side simply as $-\left(1+\partial C^{F} / \partial B\right)$ as in $(26)$.

${ }^{16}$ Given the endogeneity of optimal tax expressions, one must exercise caution in comparative statics predictions (see for example Kaplow, 2007). The discussion around (44) is necessarily speculative.
} 
A sufficient condition for $\partial V^{F} / \partial p_{I I}>0$ is:

$$
\begin{gathered}
\frac{F\left[\bar{n}\left(\hat{\tau}^{F}, \hat{B}^{F}\right)\right]}{\frac{\theta\left(1-p_{I}\right)}{(1-\theta) p_{I I}}+F(\overline{\bar{n}})} \cdot\left[\frac{n f(\overline{\bar{n}})}{F(\overline{\bar{n}})}\right]\left[\frac{\hat{C}^{F} \overline{\bar{n}}_{C}}{n}\right]\left\langle 1+\frac{\hat{\tau}^{F}}{\hat{C}^{F}} y\left(\overline{\bar{n}}, 1-\hat{\tau}^{F}, \hat{B}^{F}\right)\right\rangle \\
-\left\langle\left[1-\frac{F\left[\bar{n}\left(\hat{\tau}^{F}, \hat{B}^{F}\right)\right]}{F(\overline{\bar{n}})}\right]+\frac{1}{F(\overline{\bar{n}})} \frac{\hat{\tau}^{F}}{\hat{C}^{F}} \int_{\bar{n}}^{\overline{\bar{n}}} y\left(n, 1-\hat{\tau}^{F}, \hat{B}^{F}\right) d F(n)\right\rangle>0
\end{gathered}
$$

This condition resembles (29) from the fixed budget analysis, with the difference that foregone tax revenue from those with $\bar{n}\left(\hat{B}^{F}\right)<n \leq \overline{\bar{n}}\left(\hat{B}^{F}, \hat{C}^{F}\right)$ is included. Notice that the lower this foregone revenue, the lower the subtracted term in (29).

\section{Concluding Remarks}

We modelled the optimal combination of universal and categorical benefits for an economy where a fraction of individuals are unable to work, whilst those who can work differ continuously in their productivity. The categorical benefit is conditioned ex-ante on applicants being unable to work; and ex-post on recipients not working. However, the awards test makes classification errors and the no-work condition is either not enforced or fully enforced.

Our welfare results can be viewed in terms of policy reforms to an awards test. In doing so it is useful to distinguish between (i) investments which improve discriminatory power; and (ii) changes in the liability threshold which simply make the test more lenient/stringent, implying a trade-off between error types. In our framework maximum social welfare unambiguously decreases in the Type I error propensity, implying there will always be a marginal welfare gain to investments which reduce Type I errors. In reality these must be offset against the investment costs. The welfare effect of Type II errors depends importantly on the enforcement of the ex-post condition. Under No Enforcement maximum welfare unambiguously falls with the Type II errors propensity. There will thus be a marginal welfare gain to investments which lower Type II errors, whilst the effect of changes in the liability threshold will depend on the relative harm that either error type does to society. Contrastingly, under Full Enforcement there are conditions where maximum welfare may increase with the Type II error propensity. Where these conditions hold an implication is that focus should be placed on reforms which lower Type I errors. 


\section{References}

Akerlof, G. A. (1978). The Economics of "Tagging" as Applied to the Optimal Income Tax, Welfare Programs, and Manpower Planning. American Economic Review, 68:8-19.

Atkinson, A. B. (1990). Public economics and the economic public. European Economic Review, 34:225-248.

Atkinson, A. B. (1995). Public Economics in Action: The Basic Income/ Flat Tax Proposal. Oxford University Press, Oxford.

Atkinson, A. B. and Stiglitz, J. E. (1980). Lectures on Public Economics. McGraw-Hill Book Company, New York.

Beath, J., Lewis, G., and Ulph, D. (1988). Policy targeting in a new welfare framework with poverty. In Hare, P. G., editor, Surveys in Public Sector Economics, pages 161-185. Basil Blackwell, Oxford, UK.

Benitez-Silva, H., Buchinsky, M., and Rust, J. (2004). How large are the classification errors in the social security disability award process? NBER Working Paper Series, (10219).

Callan, T., O’Donoghue, C., Sutherland, H., and Wilson, M. (1999). Comparative analysis of Basic Income proposals: UK and Ireland. Number February. The Microsimulation Unit, Department of Applied Economics, University of Cambridge, Cambridge.

Currie, J. (2006). The Take-Up of Social Benefits. In Auerbach, A. J., Card, D., and Quigley, J. M., editors, Public policy and the income distribution, pages 80-148. Russel Sage.

Duclos, J.-Y. (1995). Modelling the take-up of state supoort. Journal of Public Economics, 58:391-415.

Fuller, D. L., Ravikumar, B., and Zhang, Y. (2015). Unemployment Insurance Fraud and Optimal Monitoring. American Economic Journal: Macroeconomics, 7(2):249-290.

Goodin, R. (1985). Erring on the Side of Kindness in Social Welfare Policy. Policy Sciences.

Immonen, R., Kanbur, R., Keen, M., and Tuomala, M. (1998). Tagging and Taxing: The Optimal Use of Categorical and Income Information in DesigningTax/Transfer Schemes. Economica, 65(258):179-192.

Jacquet, L. (2006). Optimal disability assistance when fraud and stigma matter. Queen's Economics Department Working Paper, (1098). 
Jacquet, L. (2014). Tagging and redistributive taxation with imperfect disability monitoring. Social Choice and Welfare, 42(2):403-435.

Kaplow, L. (2007). Optimal income transfers. International Tax and Public Finance, 14:295325.

Mirrlees, J. (1971). An Exploration in the Theory of Optimal Income Taxation. The review of economic studies, 38(2):175-208.

Moffitt, R. (1983). An economic model of welfare stigma. The American Economic Review, 73(5):1023-1035.

Nagi, S. (1969). Disability and Rehabilitation: Legal, Clinical, and Self-Concepts and Measurement. Ohio State University Press.

Parsons, D. (1996). Imperfect 'tagging' in social insurance programs. Journal of Public Economics, 62:183-207.

Salanié, B. (2002). Optimal demogrants with imperfect tagging. Economics Letters, 75:319324.

Slack, S. E. (2015). Revisiting the optimal linear income tax with categorical transfers. Economics Letters, 134:73-77.

Slack, S. E. (2016). Revisiting the Optimal Linear Income Tax with Categorical Transfers. University of St Andrews PhD Thesis, Chapter 3.

Stern, N. (1982). Optimum taxation with errors in administration. Journal of Public Economics, 17:181-211.

Van Parijs, P. (2004). Basic Income: A Simple and Powerful Idea for the Twenty-First Century. Politics \& Society, 32(1):7-39.

Viard, A. (2001a). Optimal Categorical Transfer Payments: The Welfare Economics of Limited LumpSum Redistribution. Journal of Public Economic Theory, 3(December 2000):483-500.

Viard, A. (2001b). Some Results on the Comparative Statics of Optimal Categorical Transfer Payments. Public Finance Review, 29(2):148-180.

Yaniv, G. (1986). Fraudulent collection of unemployment benefits: A theoretical analysis with reference to income tax evasion. Journal of Public Economics, 30. 